\title{
Diversity and distribution of Orthoptera communities of two adjacent mountains in northern part of the Carpathians
}

\author{
Anton Krištín ${ }^{1}$, Benjamín Jarčuška ${ }^{1}$, Peter Kaňuch ${ }^{1}$ \\ 1 Institute of Forest Ecology SAS, L. Štúra 2, Zvolen, SK-96053, Slovakia \\ Corresponding author: Anton Krištín (kristin@ife.sk)
}

Received 19 November 2019 | Accepted 24 December 2019 | Published 31 December 2019

Citation: Krištín A, Jarčuška B, Kaňuch P (2019) Diversity and distribution of Orthoptera communities of two adjacent mountains in northern part of the Carpathians. Travaux du Muséum National d'Histoire Naturelle “Grigore Antipa” 62(2): 191-211. https://doi.org/10.3897/travaux.62.e48604

\begin{abstract}
During 2013-2017, assemblages of bush-crickets and grasshoppers were surveyed in two neighbouring flysch mountains - Čergov Mts (48 sites) and Levočské vrchy Mts (62 sites) - in northern part of Western Carpathians. Species were sampled mostly at grasslands and forest edges along elevational gradient between 370 and $1220 \mathrm{~m}$ a.s.l. Within the entire area $\left(\mathrm{ca} 930 \mathrm{~km}^{2}\right)$ we documented 54 species, representing 38\% of Carpathian Orthoptera species richness. We found the same species number (45) in both mountain ranges with nine unique species in each of them. No difference in mean species richness per site was found between the mountain ranges (mean $\pm \mathrm{SD}=12.5 \pm 3.9$ ). Elevation explained $2.9 \%$ of variation in site species richness. Elevation and mountain range identity explained $7.3 \%$ of assemblages composition. We found new latitudinal as well as longitudinal limits in the distribution for several species. Occurrence and distributional patterns of some noteworthy species (Isophya stysi, Pholidoptera transsylvanica, Poecilimon schmidtii, Polysarcus denticauda, Pseudopodisma nagyi, Chorthippus tatrae), as well as habitat specialists (Chorthippus pullus, Tetrix tuerki) are discussed.
\end{abstract}

\section{Keywords}

grasshoppers, bush-crickets, zoogeography, ecology, Central Europe 


\section{Introduction}

The Carpathian Mountains are the largest mountain range in Central and Southeastern Europe and an important center of endemism and biodiversity for many plant and animal groups (Ronikier 2011, Mráz and Ronikier 2016, Jarčuška et al. 2019). The northern part of Carpathian Mts has been less studied area for distributional data of Carpathian Orthoptera untill 2000 (Mařan 1956, Bazyluk and Liana 2000, Jarčuška et al. 2015). Today we know that several species and even genera of Orthoptera have the northernmost range limit there (Hochkirch et al. 2016). For example, two species of Pseudopodisma (Galvagni and Fontana 1996), then Poecilimon schmidtii, Isophya stysi and Pholidoptera transsylvanica (Krištín and Kaňuch 2013, 2019, Jarčuška et al. 2015, Krištín and Jarčuška 2016, Krištín et al. 2019).

The northern part of Western Carpathians in Slovakia and southern Poland is characterized by cooler climate, different types of grasslands and forest habitats along elevational gradients up to $2655 \mathrm{~m}$ a.s.l. Relatively diverse environmental conditions may explain rich species diversity of insect and particularly Orthoptera assemblages (Gavlas et al. 2007, Krištín 2010). Grassland habitats, broadleaved forests and their ecotones are relatively well preserved there, being a regional hotspot for central European orthopteran fauna (Bazyluk 1971, Bazyluk and Liana 2000, Nagy 2005, Kenyeres et al. 2009, Krištín and Iorgu 2014, Jarčuška et al. 2015). Several Orthoptera species have been described there (e.g. Isophya pienensis, Isophya posthumoidalis, Chorthippus tatrae, Isophya fatrensis, Chorthippus smardai - Mařan 1954, Bazyluk 1971, Chládek and Harz 1983, Chládek 2007, Chládek 2014) and some of these Carpathian endemics are expected to have significant populations there (Jarčuška et al. 2015, Krištín and Iorgu 2014, Chládek 2014, Hochkirch et al. 2016). Elevational patterns in diversity of taxa may be apparent on a range of spatial scales (Gaston and Williams 1996). At the finest scale the number of insect species associated with a particular habitats may decline with rising elevation (Hodkinson 2005). The distributions of many terrestrial organisms are currently shifting in latitude or elevation in response to changing climate (Hickling et al. 2006, Chen et al. 2011). Therefore, regularly updated knowledge about the range boundaries is important for understanding the effects of climate change on species distributional patterns (Keppel et al. 2012, Fournier et al. 2017), especially in mountain systems (Schmitt 2009, Ronikier 2011).

In this study we analyzed species composition and abundance of Orthoptera assemblages in Čergov Mts and Levočské vrchy Mts in relationship to the elevation and we discussed the importance of these two mountain ranges for Carpathian Orthoptera diversity. 


\section{Material and methods}

\section{Study area and sites}

Species assemblages were studied in two Carpathian mountains (Čergov Mts, ca $301 \mathrm{~km}^{2}-48$ sites and Levočské vrchy Mts ca $625 \mathrm{~km}^{2}-62$ sites) (Fig. 1). Altogether 110 sites were sampled along an elevational gradient ranging from 370 to 1220 $\mathrm{m}$ a.s.l. (49.0274-49.3381 $\mathrm{N}, 20.38074-21.23336^{\circ} \mathrm{E}$; Appendix 2, 3). Most of the study area is located on flysch bedrock, with similar elevational gradients in both mountains (Čergov $1157 \mathrm{~m}$ a.s.l., Levočské vrchy Mts $1289 \mathrm{~m}$ a.s.l.). Both mountain ranges are mostly forested landscapes. Levočské vrchy Mts are covered more with secondary coniferous forests and influenced by cold climate of High Tatra Mts from the west while Čergov Mts. are predominantly covered with broadleaved forests influenced by warm climate of Pannonian area from the southeast. Following these characteristics, we studied substantially more sites in adjacent coniferous forests in Levočské vrchy Mts (48.4 vs $8.3 \%$ in Čergov Mts.) and on other hand more sites in broadleaved forests in Čergov Mts (56.3\% vs $11.2 \%$ in Levočské vrchy Mts), with similar ratio of mixed and azonal forests (Appendix 2,3).

\section{Data collection}

Field survey was carried out mostly in June - August in 2013-2017 (4 sites in 2009), when 24 of 110 study sites (12 in each mountain range) were visited repeat-

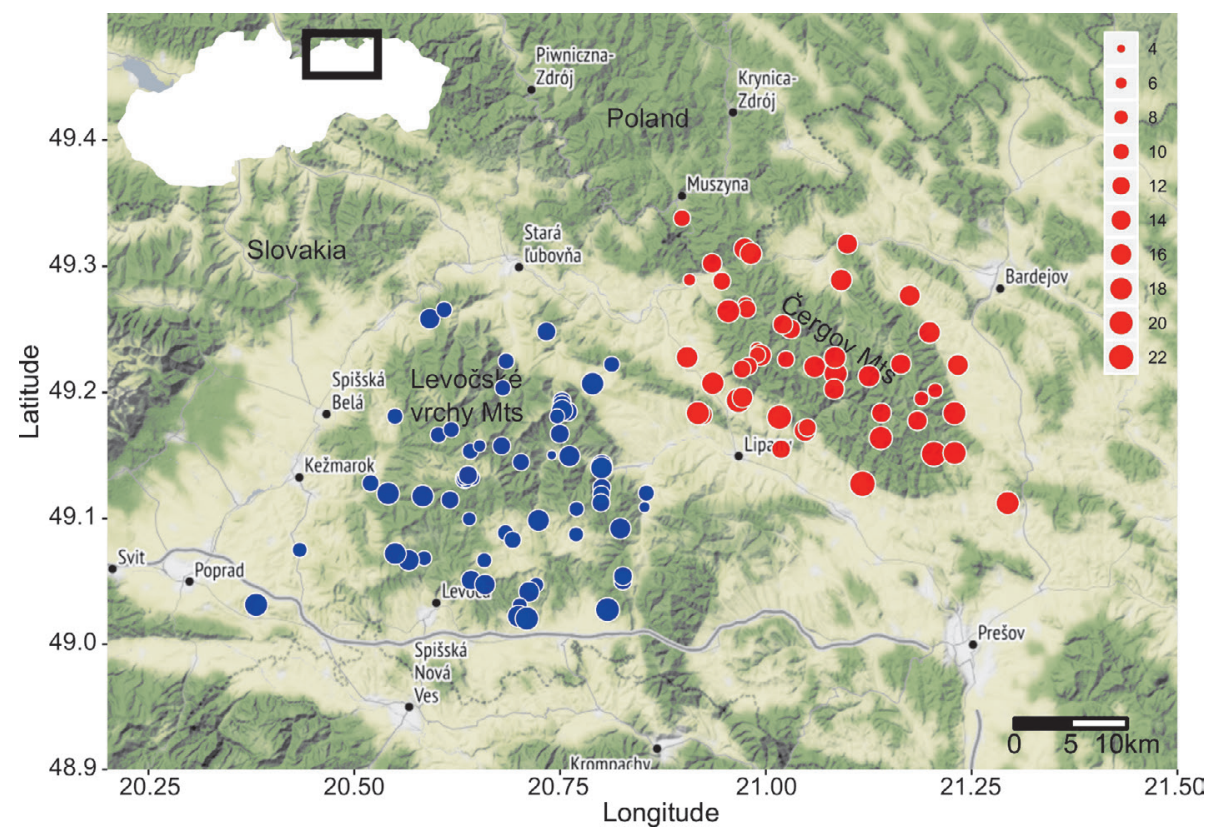

Figure 1. Species richness (circles) in Levočské vrchy (62 sites; blue) and Čergov Mts (48 sites; red) in northern Carpathians and their location within Slovakia and Poland. 
edly (up to 3 visits, Appendix 2,3). The material was sampled mostly by sweeping herb and shrub vegetation by three or four persons (ca. 2000 sweeps per site). This method was supplemented with acoustical identification, beating from lower part of trees and shrubs and individual collection of specimens in characteristic habitats of the site. We spent at least two hours of collection at each site. Abundance of individual species on the site was expressed by using the following classification scale: 1 - very rare (less than 3 adult specimens), 2 - rare (3-10 specimens), 3 - abundant (11-100 specimens), 4 - very abundant (more than 100 specimens). Relative semiquantitative values of abundance listed in the results represent the highest recorded values of the adults per visit corresponding to one site (Appendix 4, 5).

Specimens were identified directly in the field or fixed using $75 \%$ ethanol and determined in the lab using identification keys (Harz 1969, 1975, Heller et al. 2004). The system and the nomenclature was used according to Cigliano et al. (2019), the geographical data about the origin and distribution follow the work by Harz (1969, 1975), Ingrisch and Köhler (1998) and Rácz (1998) (Appendix 1, 4, 5).

For each surveyed site, the elevation was derived from the map. Frequency of species occurrence $(\% \mathrm{~F})$ was calculated for whole dataset and for each mountain range separately.

\section{Data analysis}

Difference in number of species between two mountain ranges was tested using general linear model (GLM) with Poisson distribution and log-link function. Ensifera and Mantodea species (only for analysis purpose, single representative of Mantodea was pooled with Ensifera), Caelifera and all species were used as response variables also in separate analyses. The mountain range was used as categorical variable and elevation as continuous variable in the analysis. Function Anova from package 'car' (Fox and Weisberg 2019) with type III sum of squares and contr.sum contrasts were used to test statistical significance of variables in the GLM. Pseudo$\mathrm{R}^{2}$ was calculated using package DescTools (Signorell et al. 2019)

Statistical significance of differences in orthopteran assemblage structure (i.e semiquantitative abundance of species) between the two mountain ranges and along elevational gradient was tested using function manyglm from package 'mvabund' (Wang et al. 2019). It has been shown that generalised linear models framework is a flexible powerful framework for analysing abundance data and have greater power than distance-based community methods (e.g. used in ANOSIM and SIMPER procedures; Wang et al. 2012). A multivariate linear model with mountain range, elevation and interaction between them as explanatory variables was fitted with negative binomial distribution of data. Variation in multivariate response explained by an explanatory variable in the model $-\mathrm{R}^{2}$ - was calculated using the package's function best.r.sq. Subsequently, species with the greatest contribution to the dissimilarity observed between mountains and along elevational gradient were identified. All analyses were performed in R environment (R Core Team 2019). 


\section{Results}

A total number of 54 Orthoptera (24 Ensifera and 30 Caelifera) and one Mantodea species were detected across 110 studied sites in both studied mountains (Appendix $1,4,5$ ). In spite of different number of sampling sites (48 Čergov, 62 Levočské vrchy Mts.) and twice larger area of Levočské vrchy mountain range, we found the same species number (45) in both areas with nine unique species in each of them (Appendix 1, 4, 5, Fig. 1). However, the mean $( \pm S D)$ number of Orthoptera and Mantodea species per site was $14.3( \pm 3.76)$ and $11.2( \pm 3.61)$ in Čergov and Levočské vrchy Mts, respectively (Fig. 2). No significant differences in number of all species together and also number of Ensifera and Mantodea species had been observed between two mountain ranges. Only the elevation affected species richness and the pattern of association between elevation and species richness was the same for both mountain ranges (Table 1).

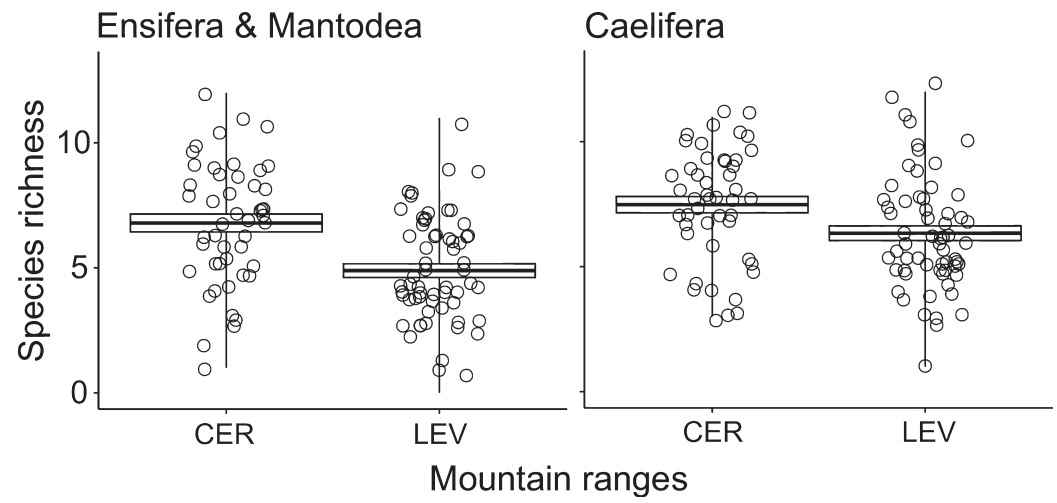

Figure 2. Species richness of Ensifera and Mantodea (left) and Caelifera (right) in two studied mountains. Means, \pm SE (box) and range (min-max; whiskers) are presented.

Table 1. Outputs of GLM with Poisson distribution with log link function, modelling number of species, Ensifera and Mantodea, and Caelifera separately using mountain range and elevation as explanatory variables.

\begin{tabular}{lllllll}
\hline & All species & \multicolumn{5}{c}{$\begin{array}{l}\text { Ensifera, } \\
\text { Mantodea }\end{array}$} \\
\hline & $\mathrm{F}_{1,106}$ & $\mathrm{p}$ & $\mathrm{F}_{1,106}$ & $\mathrm{p}$ & $\mathrm{F}_{1,106}$ & $\mathrm{p}$ \\
Mts & 0.142 & 0.707 & 0.088 & 0.768 & 0.076 & 0.784 \\
elevation & 7.425 & $\mathbf{0 . 0 0 8}$ & $\mathbf{0 . 0 8 8}$ & $\mathbf{0 . 0 3 9}$ & $\mathbf{4 . 7 3 6}$ & $\mathbf{0 . 0 3 2}$ \\
$\begin{array}{l}\text { Mts } \\
\text { elevation }\end{array}$ & 0.284 & 0.595 & 4.356 & 0.522 & 0.039 & 0.845 \\
Pseudo-R & 0.045 & & 0.042 & & 0.018 & \\
\hline
\end{tabular}


Mean number of both Ensifera/Mantodea and Caelifera species per site was higher in Čergov Mts (further CER) than Levočské vrchy Mts (further LEV) - 6.8 $(\mathrm{SD}=2.47)$ vs $4.9(2.16)$ and $7.5(2.25)$ vs $6.3(2.30)$ for Ensifera and Caelifera, respectively (Fig. 2).

Elevation was negatively associated with number of species in both mountain ranges, however association was weak (Fig. $3, \mathrm{R}^{2}=0.029, \mathrm{P}<0.001$ ).

The multivariate linear model revealed that the Orthoptera and Mantodea assemblages composition were affected by mountain range (ANOVA, $\mathrm{df}=1,108$, likelihood-ratio test $(\mathrm{LRT})=260.2, \mathrm{P}=0.001)$, and elevation $(\mathrm{df}=1,107, \mathrm{LRT}=246.3$, $\mathrm{P}=0.001$ ). Interaction between mountain range and elevation was not statistically significant $(\mathrm{df}=1,101, \mathrm{LRT}=50.7, \mathrm{P}=0.052)$. The model variables explained $7.3 \%$ of variability in assemblages' composition (elevation and mountain range explained $4.5 \%$ and $3.9 \%$, respectively).

Species with the greatest contribution to the dissimilarity observed in species abundance between two mountain ranges (CER and LEV) were Pholidoptera transsylvanica $(\mathrm{LRT}=55.619, \mathrm{P}=0.001 ; \% \mathrm{~F} \mathrm{CER} / \mathrm{LEV}=50 / 0 \%)$, Isophya camptoxypha $(\mathrm{LRT}=18.425, \mathrm{P}=0.003 ; \% \mathrm{~F}=0 / 23 \%)$, Chorthippus tatrae $(\mathrm{LRT}=$ $11.109, \mathrm{P}=0.026 ; \% \mathrm{~F}=0 / 15 \%)$, Omocestus viridulus ( $\mathrm{LRT}=12.735, \mathrm{P}=0.010 ; \% \mathrm{~F}$ $=46 / 76 \%)$, Stenobothrus lineatus $(\mathrm{LRT}=10.57, \mathrm{P}=0.038 ; \% \mathrm{~F}=40 / 13 \%$, Decticus verrucivorus $(\mathrm{LRT}=10.545, \mathrm{P}=0.038 ; \% \mathrm{~F}=79 / 63 \%)$, less also Phaneroptera falcata $(\mathrm{LRT}=9.508, \mathrm{P}=0.054, \% \mathrm{~F}=39.6 / 9.7)$, Poecilimon schmidtii $(\mathrm{LRT}=8.763, \mathrm{P}=$ $0.076, \% \mathrm{~F}=10.4 / 0)$ and Platycleis grisea $(\mathrm{LRT}=8.685, \mathrm{P}=0.076, \% \mathrm{~F}=10.4 / 0)$.

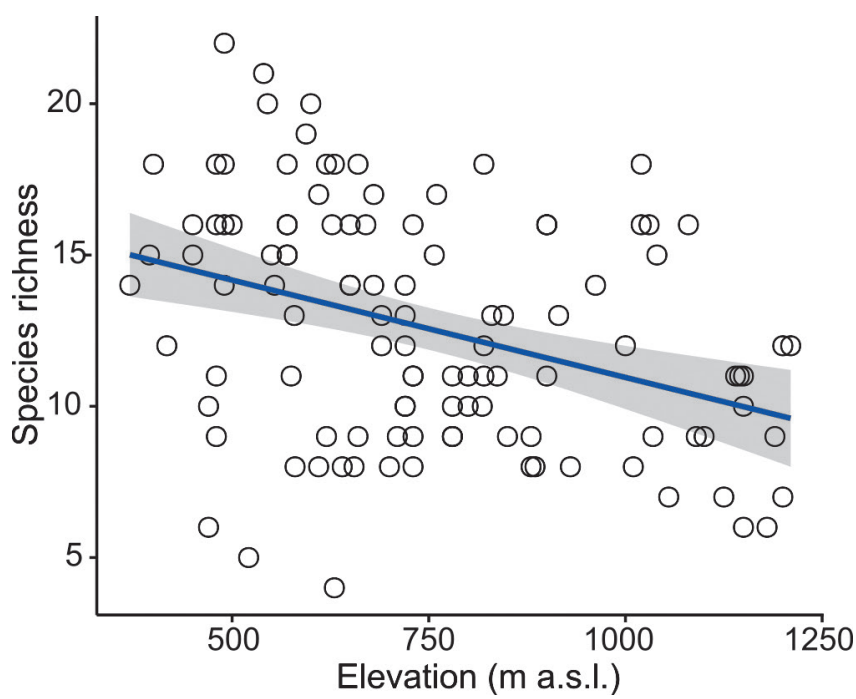

Figure 3. Association between species richness of Orthoptera and Mantodea and elevation of 110 sampled sites in Čergov Mts and Levočské vrchy Mts. Elevation explained 2.9\% of species richness in GLM model. 
The species Leptophyes albovittata $(\mathrm{LRT}=45.887, \mathrm{P}=0.001), P$. falcata $(\mathrm{LRT}=$ $28.116, \mathrm{P}=0.001), P$. transsylvanica $(\mathrm{LRT}=12.913, \mathrm{P}=0.017)$, Tetrix tenuicornis $(\mathrm{LRT}=14.416, \mathrm{P}=0.011)$, Chorthippus dorsatus $(\mathrm{LRT}=16.393, \mathrm{P}=0.004)$ differentiated assemblages in relation to elevation - all these species except $P$. transsylvanica were more frequent and abundant in lower elevations.

Several other species were distinct for both mountain ranges (Isophya stysi, Pseudopodisma nagyi for CER, Tetrix subulata for LEV, Fig. 4), having low frequency and abundance. Some other didn't have interpretation value, while occurríng in only one site (e.g. Conocephalus fuscus, Chorthippus vagans, O. caerulescens, Chorthippus oschei in CER, Pholidoptera aptera, Arcyptera fusca, Miramella alpina, in LEV, see Appendix 1, 4, 5).

\section{Discussion}

\section{Species richness}

This is the first report on Orthoptera assemblages along the Slovak-Polish borderline between SE Poland (Theuerkauf et al. 2005) / NE Slovakia (Jarčuška et al. 2015, Jarčuška 2019) and Tatra Mts (Krištín 2010) and it is filling the gap in knowledge from this part of Western Carpathians (Hochkirch et al. 2016, Jarčuška et al.

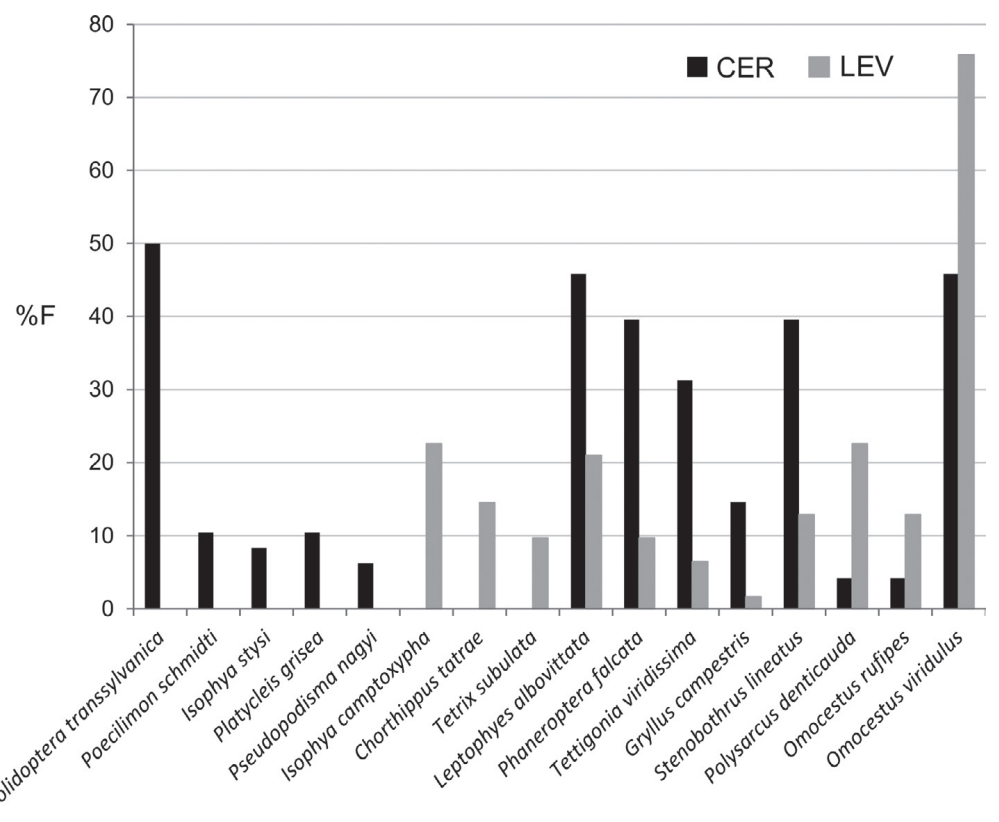

Figure 4. Frequency of eight most distinct species and other 8 selected Orthoptera species with substantialy different frequencies ( $>40 \%$ difference, right columns) in the two studied mountains. Ten distinct species with only one site occurrence are not shown. 
2019). Altogether 142 Orthoptera species were found in Carpathians (Krištín and Iorgu 2014). A negative latitudinal trend in species richness in Carpathian Mts from 91 species $/ 100 \mathrm{~km}^{2}$ grid cell in the south to 47 species/cell in the northwest (Jarčuška et al. 2019) was registered. We found 54 Orthoptera species, i.e. 38\% out of 142 species known from the Carpathian Mts (Krištín and Iorgu 2014) in both studied mountain ranges $\left(\mathrm{ca} 930 \mathrm{~km}^{2}\right.$ ). For comparison, in the adjacent Tatra Mts and NE Slovak/SE Polish Carpathians a similar species richness was found, i.e. 45 species in Tatra Mts (Krištín 2010), and 54 species in NE Slovak/SE Polish Carpathians (Theuerkauf et al. 2005, Jarčuška et al. 2015, Jarčuška 2019). Slightly higher numbers of species were found in mountain ranges with similar area $\left(\mathrm{km}^{2}\right)$ but located southward from the study area, e.g. 67 species in Cserhát Mts. in N Hungary (Szövényi et al. 2013), 60 species in Börzsöny Mts. (Erdélyi et al. 2017) or 56 in Polana Mts., Central Slovakia (Krištín and Hrúz 2005). Each one of these areas has a characteristic species composition, dependant on the distance from the Pannonian basin. Although the study area does not represent a hot spot for Orthoptera biodiversity in Europe, we found two species of European importance there (P. transsylvanica and I. stysi).

\section{Differences of species assemblages in two mountain ranges}

Dissimilarity in species composition between the two studied mountain ranges ( $20 \%$ of species in each mountain range $=9$ ), could be explained by their different position and distance from the Pannonian basin and High Tatra Mts, by cooler climate in Levočské vrchy Mts and warmer climate in Čergov Mts. Three once believed south-eastern Carpathian and Pontomediterranean species $P$. transsylvanica ( $\mathrm{F}=$ $50 \%$ of 48 sites), P. schmidtii (10.4\%), I. stysi (8.3\%), were found only in Čergov Mts. These mountains were found to be the nortwesternmost boundary of their global range that follows relatively continual distribution from the southeast (Hochkirch et al. 2016). This knowledge supports the results of quantitative regionalization of the Carpathians based on Orthoptera, that these south-eastern Carpathian species are distributed more westward from the former outlined boundary between Western and Eastern Carpathians (Jarčuška et al. 2019). Furthermore, some thermophilous species were found in Čergov Mts mostly in significantly higher frequencies and abundance ( $P$. grisea, L. albovittata, $P$. falcata, Fig. 4$)$ than in cooler Levočské vrchy Mts. Only in Levočské vrchy Mts we found distinct Western Carpathian endemic Ch. tatrae (on 15\% of 62 sites, having the easternmost range limit there), or mountainous species I. camptoxypha (23\%), and other mountainous species found in lower frequencies or accessoric, but having the eastern and northern range limit there (Miramella alpina, Arcyptera fusca; Hochkirch et al. 2016). Furthermore, we found several species with significantly different frequencies and abundances such as mountainous species Omocestus viridulus and Polysarcus denticauda dominating in Levočské vrchy Mts and P. nagyi in Čergov Mts, which is the northernmost boundary of its fragmented range in Europe (Hochkirch et al. 2016). Habitat specialists such as Chorthippus pullus and Tetrix tuerki were found only in azonal posi- 
titions of gravel banks along creeks on the piedmont in both mountain ranges (in the Torysa and Poprad river catchments), also the study area belongs to the strongholds of these species in Western Carpathians. Carpathian Dancing Grasshopper C. oschei (single male) was found only in one site and only in the Čergov Mts, together with more abundant and related C. albomarginatus (> 10 specimens). This record may widen our knowledge on the hybrid zone of these two species, but it needs further research.

\section{Elevational patterns}

In this small scale study (ca $930 \mathrm{~km}^{2}$ of both mountains) we confirmed general pattern of negative correlation between the species richness and increasing elevation (e.g. Hodkinson 2005, Nufio et al. 2010, Fournier et al. 2017). Only or mostly in higher elevations (> $900 \mathrm{~m}$ a.s.l.) Metrioptera brachyptera, P. aptera, M. alpina, A. fusca were found. As expected, we found thermophilous species such as I. stysi, Platycleis grisea, Poecilimon intermedius, Ch. oschei, Stenobothrus crassipes mostly or only in lower elevations ( $<500 \mathrm{~m}$ a.s.l.).

\section{Acknowledgements}

The research was supported by the Grant Agency VEGA (grants no. 2/0097/16 and 2/0076/19). The authors are grateful to Peter Tuček, Elena and Ionuț Iorgu, who helped with field collections.

\section{References}

Bazyluk W (1971) Prostoskrzydłe (Orthoptera) Biesczadów Zachódnich wraz z opisem Isophya posthumoidalis n.sp. [Grasshoppers and crickets (Orthoptera) of the Biesczady Zachódne with the description of Isophya posthumoidalis n.sp.]. Fragmenta Faunistica 22: 127-159. [in Polish]

Bazyluk W, Liana A (2000) Prostoskrzydłe (Orthoptera). [Grasshoppers and crickets (Orthoptera)]. Katalog fauny Polski 17 (2): 1-156. [in Polish]

Cigliano MM, Braun H, Eades DC, Otte D (2019) Orthoptera Species File. Version 5.0/5.0. http://Orthoptera.SpeciesFile.org. Accessed 16 October 2019.

Chen IC, Hill JK, Ohlemuller R, Roy DB, Thomas CD (2011) Rapid range shifts of species associated with high levels of climate warming. Science 333: 1024-1026.

Chládek F (2007) Isophya fatrensis sp. nov. aus der Slowakei (Orthoptera, Ensifera). [Isophya fatrensis sp. nov. from Slovakia (Orthoptera, Ensifera)]. Tetrix 2(3): 9-12. [in German]

Chládek F (2014) Chorthippus smardai - eine neue Art aus der Nordslowakei (Orthoptera s.l., Caelifera, Acrididae). [Chorthippus smardai - new species from Northern Slovakia (Orthoptera s.l., Caelifera, Acrididae)]. Tetrix 2(10): 37-40. 
Chládek F, Harz K (1983) Chorthippus tatrae Harz ist eine gute Art. [Chorthippus tatrae Harz is a good species]. Articulata 2: 18-19.

Erdélyi A, Nagy B, Puskás G, Szövényi G (2017) The Orthoptera fauna of Börzsöny Mountains, Hungary. Articulata 32: 59-82.

Fournier A, Barbet-Massin M, Rome Q, Courchamp F (2017) Predicting species distribution combining multi-scale drivers. Global Ecology and Conservation 12: 215-226.

Fox J, Weisberg S (2019) An R companion to applied regression. $3^{\text {rd }}$ Edition. Sage Publications, Thousand Oaks.

Galvagni A, Fontana P (1996) Descrizione della Pseudopodisma nagyi specie nuova dell' Ungheria Nord-orientale (Insecta Orthoptera Catantopidae). [Description of Pseudopodisma nagyi sp. n. from NE Hungary (Insecta Orthoptera Catantopidae)]. Atti della Accademia Roveretana degli Agiati, Serie 7, 5(B) 245 (1995): 209-232. [in Italian] Gaston KJ, Williams PH (1996) Spatial patterns in taxonomic diversity. In: Gaston KJ (Ed.) Biodiversity: A Biology of Numbers and Difference. Blackwell Science, Oxford, 202-229. Gavlas V, Bednár J, Krištín A (2007) A comparative study on orthopteroid assemblages along a moisture gradient in the Western Carpathians. Biologia 62: 95-102.

Harz K (1969) Die Orthopteren Europas [The Orthoptera of Europe]. Vol. I. Dr. W. Junk N.V., The Hague, 749 pp.

Harz K (1975) Die Orthopteren Europas [The Orthoptera of Europe]. Vol. II. Dr. W. Junk N.V., The Hague, 939 pp.

Heller K-G, Orci KM, Grein G, Ingrisch S (2004) The Isophya species of Central and Western Europe (Orthoptera: Tettigonioidea: Phaneropteridae). Tijdschrift voor Entomologie 147: 237-258.

Hickling R, Roy DB, Hill JK, Fox R, Thomas CHD (2006) The distributions of a wide range of taxonomic groups are expanding polewards. Global Change Biology 12: 450-455.

Hochkirch A, Nieto A, García Criado M, Cálix M, Braud Y, Buzzetti FM, Chobanov D, Odé B, Presa Asensio JJ, Willemse L, Zuna-Kratky T, Barranco Vega P, Barros F, Bushell M, Clemente ME, Cordero Tapia PJ, Correas JR, Dusoulier F, Ferreira S, Fontana P, García MD, Heller K-G, Iorgu IȘ, Ivković S, Kati V, Kleukers R, Krištín A, LemonnierDarcemont M, Lemos P, Massa B, Monnerat C, Papapavlou KP, Prunier F, Pushkar T, Roesti C, Rutschmann F, Şirin D, Skejo J, Szövényi G, Tzirkalli E, Vedenina V, Barat Domenech J, Defaut B, Fartmann T, Gomboc S, Gutiérrez-Rodríguez J, Holuša J, Illich I, Karjalainen S, Kočárek P, Korsunovskaya O, Liana A, López H, Morin D, OlmoVidal JM, Puskás G, Savitsky V, Stalling T, Tumbrinck J (2016) European red list of grasshoppers, crickets and bush-crickets. Publications Office of the European Union, Luxembourg, 86 pp.

Hodkinson ID (2005) Terrestrial insects along elevation gradients: species and community responses to elevation. Biological Reviews of the Cambridge Philosophical Society 80: 489-513.

Ingrisch S, Köhler G (1998) Die Heuschrecken Mitteleuropas. [Grasshoppers and crickets of Central Europe]. Westarp Wissenschaften, Magdeburg. [in German]

Jarčuška B (2019) Rovnokrídlovce (Orthoptera) a modlivky (Mantodea) NP Poloniny. Poznámky k výskytu druhov. [Orthopterans (Orthoptera) and mantids (Mantodea) of 
Poloniny National Park: notes on species occurrence]. In: Fulín M (Ed.) 42. Východoslovenský tábor ochrancov prírody. Štátna ochrana prírody Slovenskej republiky, Banská Bystrica, 40-44. [in Slovak]

Jarčuška B, Kaňuch P, Krištín A (2015) Orthoptera and Mantodea assemblages of East Carpathian Mts (Central Europe). Folia faunistica slovaca 20: 167-182.

Jarčuška B, Kaňuch P, Nad’o L, Krištín A (2019) Quantitative biogeography of Orthoptera does not support classical qualitative regionalization of the Carpathian Mountains. Biological Journal of the Linnean Society, blz143, https://doi.org/10.1093/biolinnean/ blz143

Kenyeres ZI, Rácz IA, Varga Z (2009) Endemism hot spots, core areas in European Orthoptera. Acta Zoologica Cracowiensis 52: 189-211.

Keppel G, Van Niel KP, Wardell-Johnson GW, Colin J, Yates CJ, Byrne M, Mucina L, Schut AGT, Hopper SD, Franklin SE (2012) Refugia: identifying and understanding safe havens for biodiversity under climate change. Global Ecology and Biogeography 21: 393-404.

Krištín A, Hrúz V (2005) Rovnokrídlovce (Orthoptera) a modlivky (Mantodea) Polany. (Orthoptera and Mantodea of Polana Mts). [Orthopterans (Orthoptera) and mantids (Mantodea) of Polana Mts]. ŠOP SR, Správa CHKO Polana, Ústav ekológie lesa SAV, Zvolen, 77 pp. [in Slovak]

Krištín A (2010) Rovnokrídlovce (Orthoptera) Tatier. [Orthoptera of Tatra Mts]. In: Chovancová B (Ed.) Encyklopédia Tatier, Baset, Praha, 465-468.

Krištín A, Iorgu IS (2014) Red List of grasshoppers, bush-crickets and crickets (Orthoptera) of the Carpathian mountains. In: Kadlečík J (Ed.) Carpathian Red List of forest habitats and species, State Nature Conservancy of the Slovak Republic, Banská Bystrica, 186-199.

Krištín A, Jarčuška B (2016) Distribution and ecology of the flightless bush-cricket Poecilimon schmidtii at its northern range margin. Biologia 71/9: 1049-1060.

Krištín A, Jarčuška B, Dorková M, Kaňuch P (2019) First record of Pholidoptera transsylvanica (Orthoptera, Tettigoniidae) in Poland. Entomological news 128, 4: 377-383.

Krištín A, Kaňuch P (2013) A review of distribution and ecology of three Orthoptera species of European importance with contributions from their recent north-western range. North Western Journal of Zoology 9(1): 185-190.

Krištín A, Kaňuch P (2019) Mapping of Orthoptera in Slovakia. http://www.orthoptera.sk. Accessed 11 November 2019.

Mařan J (1954) Rovnokřídlý hmyz (Orthoptera) státní přírodní reservace "Pieniny". [Orthoptera of the State Nature Reserve Pieniny]. Ochrana prrírody 9: 65-69. [in Czech, with English summary]

Mařan J (1956) Nástin entomogeografických poměrů Československa. [Outline of entomogeografic patterns in Czechoslovakia]. Acta faunistica entomologica Musei Nationalis Pragae 1: 3-25. [in Czech]

Mráz P, Ronikier M (2016) Biogeography of the Carpathians: evolutionary and spatial facets of biodiversity. Biological Journal of the Linnean Society 119: 528-559. 
Nagy B (2005) Orthoptera fauna of the Carpathian basin - recent status of knowledge and a revised checklist. Entomofauna Carpathica 17: 14-22.

Nufio CR, McGuire CR, Bowers MD, Guralnick RP, Moen J (2010) Grasshopper community response to climatic change: variation along an elevational gradient. PLoS ONE 5: 1969-1976.

Rácz IA (1998) Biogeographical survey of the Orthoptera fauna in Central part of the Carpathian Basin (Hungary): Fauna types and community types. Articulata 13: 53-69.

R Core Team (2019) R: A language and environment for statistical computing. R Foundation for Statistical Computing, Vienna, Austria. https://www.R-project.org/.

Ronikier M (2011) Biogeography of high-mountain plants in the Carpathians: an emerging phylogeographical perspective. Taxon 60: 373-389.

Schmitt T (2009) Biogeographical and evolutionary importance of the European high mountain systems. Frontiers in Zoology 6: 9.

Signorell A et mult. al. (2019) DescTools: Tools for descriptive statistics. R package version 0.99.29. https://cran.r-project.org/package=DescTools

Szövényi G, Harmos K, Nagy B (2013) The Orthoptera fauna of Cserhát Hills and its surroundings (North Hungary). Articulata 28 (1/2): 69-90.

Theuerkauf J, Rouys S, Grein G, Becker A (2005) New records of Orthoptera in the Bieszczady Mountains (Southeast Poland) with special regard to the genus Isophya. Fragmenta Faunistica 48: 9-14.

Wang Y, Naumann U, Wright ST, Warton DI (2012) mvabund - an R package for modelbased analysis of multivariate abundance data. Methods in Ecology and Evolution 3(3): 471-474.

Wang Y, Naumann U, Eddelbuettel D, Wilshire J, Warton DI, Byrnes J, Dos Santos Sylva R, Niku J, Renner I, Wright ST (2019) Package 'mvabund'. Statistical Methods for Analysing Multivariate Abundance Data. https://cran.r-project.org/package=mvabund. 
Appendix 1. Frequency (\%) of 55 Orthoptera and Mantodea species in Čergov / Levočské vrchy mountains ( $\mathrm{n}=48$ sites for Čergov Mts, $\mathrm{n}=62$ sites for Levočské vrchy Mts.; 18 distinct species for mountain range are in bold).

Barbitistes constrictus Br. v. Wattenwyl, 1878: 14.6/9.7; Conocephalus fuscus (Fabricius, 1793): 2.1/0; Decticus verrucivorus (Linnaeus, 1958): 79.2/62.9; Ephippiger ephippiger (Serville, 1831): 0/1.6; Isophya camptoxypha (Fieber, 1853): 0/22.6; Isophya kraussii Brunner von Wattenwyl, 1878: 6.3/1.6; Isophya pienensis Mařan, 1954: 39.6/29; Isophya stysi Čejchan, 1957: 8.3/0; Leptophyes albovittata (Kollar, 1833): 45.8/21; Meconema thalassinum (Degeer, 1773): 6.3/8.1; Bicolorana bicolor (Philippi, 1830): 22.9/12.9; Metrioptera brachyptera (Linnaeus, 1758): 18.8/19.4; Roeseliana roeselii (Hagenbach, 1822): 93.8/93.5; Phaneroptera falcata (Poda, 1761): 39.6/9.7; Pholidoptera aptera (Fabricius, 1793): 0/1.6; Pholidoptera griseoaptera (Degeer, 1773): 91.7/79; Pholidoptera transsylvanica (Fischer, 1853): 50/0; Platycleis grisea (Fabricius, 1781): 10.4/0; Poecilimon intermedius (Fieber, 1853): 0/1.6; Poecilimon schmidti (Fieber, 1853): 10.4/0; Polysarcus denticauda (Charpentier, 1825): 4.2/22.6; Tettigonia cantans (Fussli, 1775): 89.6/83.9; Tettigonia viridissima Linnaeus, 1758: 31.3/6.5; Gryllus campestris Linnaeus, 1758: 14.6/1.6; Tetrix bipunctata (Linnaeus, 1758): 2.1/2.7; Tetrix subulata (Linnaeus, 1758): 0/9.7; Tetrix tenuicornis Sahlberg, 1893: 12.5/6.5; Tetrix tuerki (Krauss, 1876): 4.2/4.8; Tetrix undulata (Sowerby, 1806): 4.2/3.2; Arcyptera fusca (Pallas, 1773):0/1.6; Chorthippus albomarginatus (Degeer, 1773): 12.5/3.2; Chorthippus apricarius (Linnaeus, 1758): 79.2/71; Chorthippus biguttulus (Linnaeus, 1758): 54.2/35.5; Chorthippus brunneus (Thunberg, 1815): 45.8/16.1 ; Chorthippus dorsatus (Zetterstedt, 1821): 62.5/41.9; Pseudochorthippus montanus (Charpentier, 1825): 20.8/21; Chorthippus oschei Helversen, 1985: 2.1/0; Pseudochorthippus parallelus (Zetterstedt, 1821): 91.7/85.5; Chorthippus pullus (Phillipi, 1830): 2.1/4.8; Chorthippus tatrae (Harz, 1971):0/14.5; Chorthippus vagans (Eversmann, 1848): 2.1/0; Chrysochraon dispar (Germar, 1834): 95.8/75.8; Euthystira brachyptera (Ocskay, 1826): 100/97; Gomphocerippus rufus (Linnaeus, 1758): 35.4/19.4; Mecostethus parapleurus (Hagenbach, 1822): 4.2/0; Miramella alpina (Kollar, 1833): 0/1.6; Oedipoda caerulescens (Linnaeus, 1758): 2.1/0; Omocestus haemorrhoidalis (Charpentier, 1825): 14.6/4.8; Omocestus rufipes (Zetterstedt, 1821): 4.2/12.9; Omocestus viridulus (Linnaeus, 1758): 45.8/75.8; Pseudopodisma nagyi Galvagni et Fontana, 1996: 6.3/0; Stenobothrus crassipes (Charpentier, 1825): 0/1.6; Stenobothrus lineatus lineatus (Panzer, 1796): 39.6/12.9; Stenobothrus stigmaticus (Rambur, 1838): 0/1.6; Mantis religiosa (Linnaeus, 1758):

2.1/1.6 
Appendix 2. The sampling locations in Čergov Mts.

\begin{tabular}{|c|c|c|c|c|c|c|c|}
\hline$\dot{z}$ & Locality & Habitat characteristic & \begin{tabular}{|c|} 
Adjacent \\
forest
\end{tabular} & G & Ps & Altitude & \\
\hline & & & 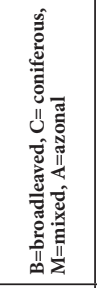 & $\mathrm{z}$ & 띠 & m a.s.l. & 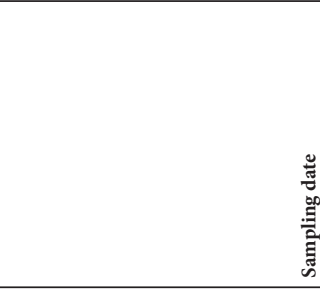 \\
\hline 1 & Muszyna (PL) & $\begin{array}{l}\text { xeric forest steppe with mixed } \\
\text { forest }\end{array}$ & M & 49.3381 & 20.8981 & 470 & 23.07.2015 \\
\hline 2 & Dubne I (PL) & mezic grasslands in mixed forest & M & 49.3139 & 20.9742 & 627 & $1.07 .2016+21.07 .2016+12.07 .2017$ \\
\hline 3 & Dubne II (PL) & mezic grasslands in mixed forest & M & 49.3101 & 20.9819 & $660-680$ & 12.07.2017 \\
\hline 4 & Leluchow (PL) & $\begin{array}{l}\text { unmown meadows, mixed forest } \\
\text { edges }\end{array}$ & M & 49.3024 & 20.9347 & 579 & $21.07 .2016+12.07 .2017$ \\
\hline 5 & Mlynská & gravel banks near the village & A & 49.3178 & 21.0991 & 370 & 31.07 .2013 \\
\hline 6 & Čirč- Manková 1 & $\begin{array}{l}\text { forest meadow in beech-spruce } \\
\text { forest }\end{array}$ & $\mathrm{C}$ & 49.2699 & 20.9755 & 780 & 01.07 .2016 \\
\hline 7 & Čirč - Manková 2 & mesic grassland in beech forest & B & 49.2662 & 20.9771 & 730 & $1.07 .2016+2.08 .2017$ \\
\hline 8 & $\begin{array}{l}\text { Čirč - Soliská creek } \\
\text { bank }\end{array}$ & $\begin{array}{l}\text { gravel bank with Myricaria in } \\
\text { beech forest }\end{array}$ & A & 49.2645 & 20.9545 & $610-620$ & $1.07 .2016+2.08 .2017$ \\
\hline 9 & $\begin{array}{l}\text { Slatina Šarišské } \\
\text { Jastrabie }\end{array}$ & bog and wetland & A & 49.2279 & 20.9043 & 570 & 30.07 .2009 \\
\hline 10 & $\begin{array}{l}\text { Bradlo range (Kyjov } \\
\text { South) }\end{array}$ & xeroterm forest steppes & M & 49.2073 & 20.9356 & 610 & $30.07 .2009+28.09 .2015$ \\
\hline 11 & $\begin{array}{l}\text { Ruská vola nad } \\
\text { Popradom }\end{array}$ & $\begin{array}{l}\text { wet grassland and beech fir spruce } \\
\text { forest edge }\end{array}$ & B & 49.2883 & 20.9465 & $550-600$ & 21.07 .2016 \\
\hline 12 & Minčol Mt. top & grasslands on the mountain top & B & 49.2345 & 20.9893 & $1100-1150$ & 28.09.2015 \\
\hline 13 & Minčol Mt. SW & $\begin{array}{l}\text { grasslands in beech forest SW } \\
\text { of the top }\end{array}$ & B & 49.2295 & 20.9934 & $1050-1120$ & 28.09 .2015 \\
\hline 14 & Minčol Mt. W & $\begin{array}{l}\text { grasslands in beech forest } \mathrm{W} \text { of } \\
\text { the top }\end{array}$ & B & 49.2298 & 20.9899 & 1035 & 28.09 .2015 \\
\hline 15 & $\begin{array}{l}\text { Sokolia dolina valleay } \\
800\end{array}$ & mezic grassland in beech forest & B & 49.2209 & 20.9788 & $790-800$ & 28.09.2015 \\
\hline 16 & $\begin{array}{l}\text { Sokolia dolina valley } \\
730\end{array}$ & xeric edge of beech spruce forest & M & 49.2185 & 20.9711 & 730 & 28.09 .2015 \\
\hline 17 & Dvoriská & $\begin{array}{l}\text { montane meadows in beech } \\
\text { forest, Vaccinium }\end{array}$ & B & 49.2205 & 21.0593 & 1020 & 31.07 .2013 \\
\hline 18 & Čergov - sedlo Priehyby & montane meadows in beech forest & B & 49.2149 & 21.0848 & $815-820$ & $31.07 .2013+14.07 .2014+13.07 .2017$ \\
\hline 19 & Solisko - Velká Javorina & $\begin{array}{l}\text { montane meadows, moor in beec } \\
\text { forest, }\end{array}$ & B & 49.2130 & 21.1254 & $1000-1056$ & 02.08 .2017 \\
\hline 20 & Lukov & pastures in mixed forests & M & 49.2892 & 21.0915 & 490 & 31.07 .2013 \\
\hline 21 & Livov & edges of beech fir forest + pastures & B & 49.2278 & 21.0840 & 650 & 31.07 .2013 \\
\hline 22 & Livovská Huta 1 & mezic meadow in beech forest & B & 49.2503 & 21.0302 & 720 & 02.08 .2017 \\
\hline 23 & Livovská Huta south & $\begin{array}{l}\text { small meadow in beech spruce } \\
\text { forest }\end{array}$ & M & 49.2263 & 21.0244 & 818 & 13.07.2017 \\
\hline 24 & Livovská Huta north & $\begin{array}{l}\text { small meadow in beech spruce } \\
\text { forest }\end{array}$ & B & 49.2541 & 21.0206 & 830 & $13.07 .2017+2.08 .2017$ \\
\hline 25 & Kríže & $\begin{array}{l}\text { grasslands (ski slopes) in mixed } \\
\text { forest }\end{array}$ & M & 49.2225 & 21.1640 & $600-700$ & 12.07.2017 \\
\hline
\end{tabular}


Appendix 2. (continued)

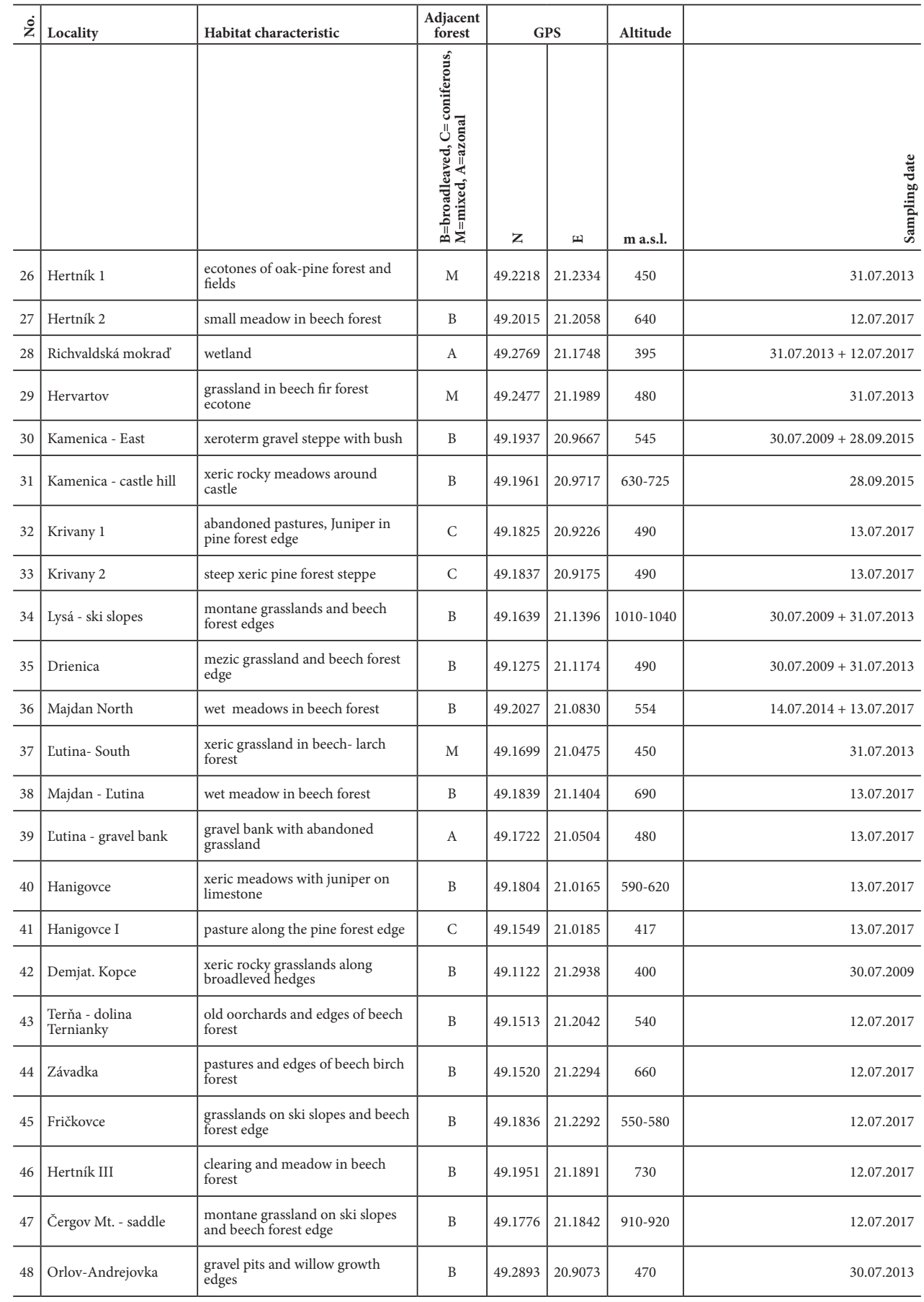


Appendix 3. The sampling locations in Levočské vrchy Mts.

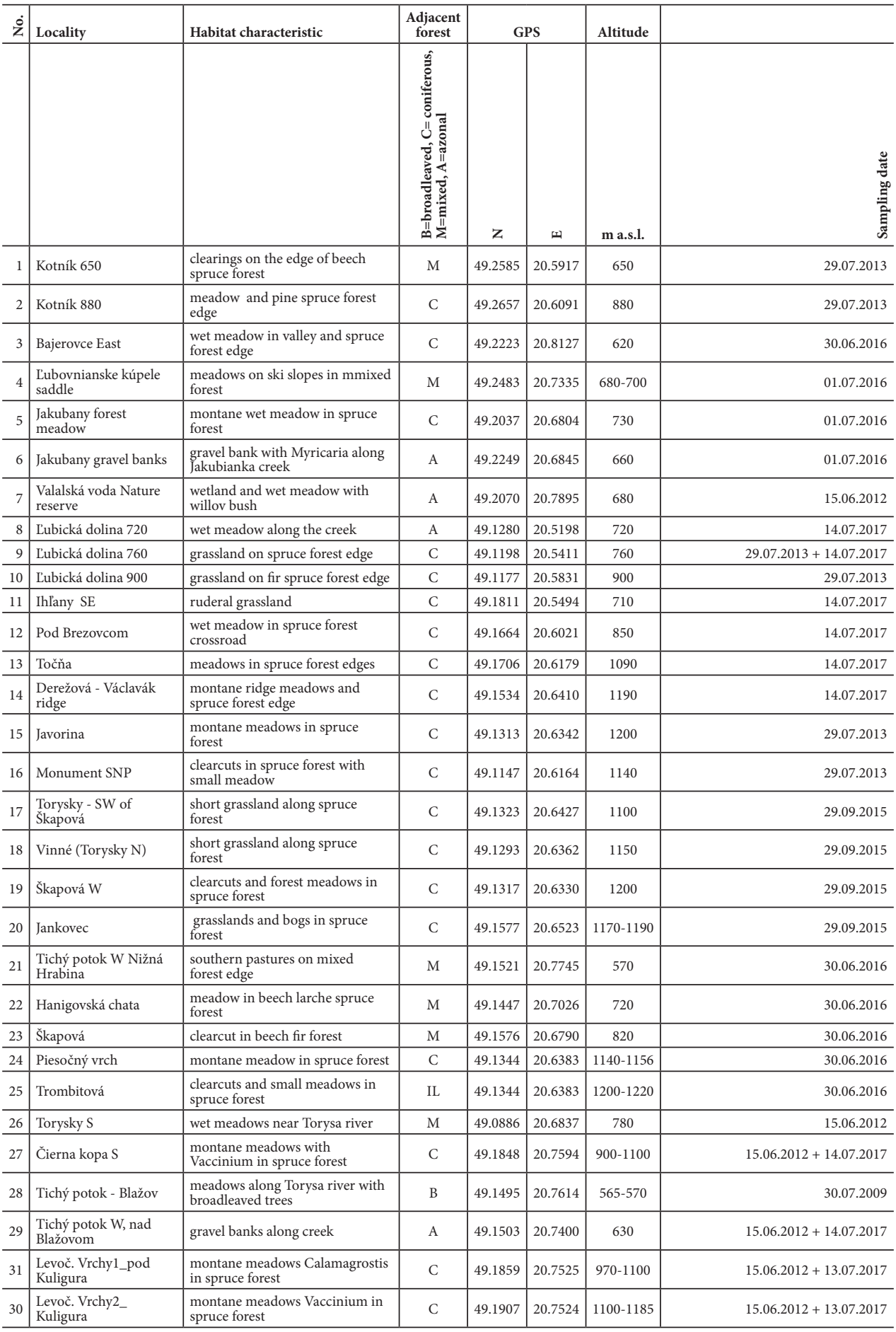


Appendix 3. (continued)

\begin{tabular}{|c|c|c|c|c|c|c|c|}
\hline \multirow[t]{2}{*}{$\dot{\mathrm{z}}$} & \multirow[t]{2}{*}{ Locality } & \multirow[t]{2}{*}{ Habitat characteristic } & \multirow[b]{2}{*}{ 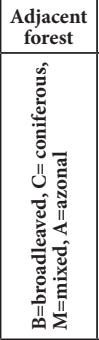 } & \multicolumn{2}{|c|}{ GPS } & \multirow[b]{2}{*}{$\begin{array}{l}\text { Altitude } \\
\\
\text { m a.s.l. }\end{array}$} & \multirow[b]{2}{*}{ 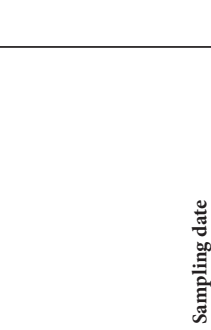 } \\
\hline & & & & z & 山 & & \\
\hline 32 & $\begin{array}{l}\begin{array}{l}\text { Levoč. vrchy3 beech } \\
\text { forest }\end{array} \\
\end{array}$ & small meadows in beech forest & B & 49.1811 & 20.7464 & $920-940$ & $15.06 .2012+13.07 .2017$ \\
\hline 33 & $\begin{array}{l}\text { Levoč. vrchy4_Stredný } \\
\text { vrch S }\end{array}$ & $\begin{array}{l}\text { wet meadow along creek in beech } \\
\text { forest }\end{array}$ & B & 49.1673 & 20.7497 & 720 & $15.06 .2012+13.07 .2017$ \\
\hline 34 & Levoč. vrchy5 Kralovec & gravel banks of Torysa river & A & 49.1499 & 20.7616 & 570 & $15.06 .2012+13.07 .2017$ \\
\hline 35 & Kuligura-Kopa & $\begin{array}{l}\text { edges of spruce forest with } \\
\text { Vaccinium, Vaccinium }\end{array}$ & $\mathrm{C}$ & 49.1932 & 20.7524 & 1145 & $20-21.07 .2013$ \\
\hline 36 & Spišské ridge & $\begin{array}{l}\text { montane meadows along larch } \\
\text { and spruce forest }\end{array}$ & $\mathrm{C}$ & 49.1075 & 20.7700 & $1000-1020$ & 29.09 .2015 \\
\hline 37 & Nižný Slavkov N & meadows along willow growths & B & 49.1089 & \begin{tabular}{|l|}
20.8527 \\
\end{tabular} & 521 & $20-21.07 .2013$ \\
\hline 38 & Brezovička S & $\begin{array}{l}\text { ruderalized meadows around } \\
\text { the road }\end{array}$ & B & 49.1201 & 20.8548 & 480 & 28.06 .2013 \\
\hline 39 & $\begin{array}{l}\text { Švábovská stráń Nature } \\
\text { reserve }\end{array}$ & $\begin{array}{l}\begin{array}{l}\text { mesophilous hay meadow in } \\
\text { spruce pine forest }\end{array} \\
\end{array}$ & $\mathrm{C}$ & 49.0313 & 20.3807 & $620-650$ & $29.07 .2013+14.07 .2014$ \\
\hline 40 & $\begin{array}{l}\text { Vrbovské mokrade } \\
\text { wetland }\end{array}$ & $\begin{array}{l}\text { wetland and wet meadows with } \\
\text { willow }\end{array}$ & B & 49.0750 & 20.4337 & 655 & 29.07.2013 \\
\hline 41 & Brinky & $\begin{array}{l}\text { montane meadows in spruce } \\
\text { forest }\end{array}$ & $\mathrm{C}$ & 49.0996 & 20.6397 & 1055 & 29.09 .2015 \\
\hline 42 & Uloža N & $\begin{array}{l}\text { montane meadows in spruce } \\
\text { forest }\end{array}$ & $\mathrm{C}$ & 49.0509 & 20.6418 & 845 & 21.07.2016 \\
\hline 43 & \begin{tabular}{|l} 
Krúžok saddle, Uloža \\
NW
\end{tabular} & $\begin{array}{l}\text { montane meadows in spruce } \\
\text { forest }\end{array}$ & $\mathrm{C}$ & 49.0474 & 20.6588 & 962 & $15.06 .2012+21.07 .2016$ \\
\hline 44 & Závada & $\begin{array}{l}\text { mown meadows on ski slopes in } \\
\text { mixed forest }\end{array}$ & M & 49.0666 & 20.6580 & $865-900$ & 29.09 .2015 \\
\hline 45 & Levočské kúpele Spa & grassland in mixed forest & $\mathrm{M}$ & 49.0683 & 20.5849 & 610 & 14.07.2017 \\
\hline 46 & Voliarňa & madow in beech oak forest & $\mathrm{B}$ & 49.0667 & 20.5662 & 757 & 14.07.2017 \\
\hline 47 & Dvorce ridge & meadows in mixed forest & $\mathrm{M}$ & 49.0720 & 20.5497 & 900 & 14.07 .2017 \\
\hline 48 & Torysky - Nižné Repaše & meadows along larch spruce forest & $\mathrm{C}$ & 49.0829 & 20.6924 & 780 & 30.06 .2016 \\
\hline 49 & Olšavica - Brutovce & meadows and ruderals along fields & $\mathrm{M}$ & 49.0873 & 20.7695 & 885 & 30.06 .2016 \\
\hline 50 & $\begin{array}{l}\text { Olšavica - Nižné } \\
\text { Repaše }\end{array}$ & grasslands in spruce forest & $\mathrm{C}$ & 49.0985 & 20.7237 & 730 & 30.07 .2009 \\
\hline 51 & Paršivá - saddle & $\begin{array}{l}\text { grasslands in saddle and spruce } \\
\text { forest edge }\end{array}$ & $\mathrm{C}$ & 49.0502 & 20.8263 & 837 & 13.07.2014 \\
\hline 52 & Bijacovce & $\begin{array}{l}\text { pastures, meadows with juniper in } \\
\text { mixed forests }\end{array}$ & M & 49.0274 & 20.8076 & 594 & 13.07.2014 \\
\hline 53 & Lúčka - forest house & meadow in mixed forest & $\mathrm{M}$ & 49.0471 & 20.7210 & 700 & 14.07 .2017 \\
\hline 54 & Jablonov N & $\begin{array}{l}\text { meadows along mixed oak pine } \\
\text { spruce forest }\end{array}$ & M & 49.0416 & 20.7123 & 720 & 14.07.2017 \\
\hline 55 & $\begin{array}{l}\begin{array}{l}\text { Jablonov N Vavrincov } \\
\text { potok }\end{array} \\
\end{array}$ & wetland along mixed forest & M & 49.0308 & 20.7010 & 580 & 14.07.2017 \\
\hline 56 & Jablonov I & $\begin{array}{l}\text { xeric meadow and adjacent } \\
\text { wetland }\end{array}$ & M & 49.0222 & 20.6994 & 500 & 14.07.2017 \\
\hline 57 & Jablonov II & $\begin{array}{l}\text { pastures along the creek near } \\
\text { village }\end{array}$ & A & 49.0203 & 20.7097 & 480 & 14.07.2017 \\
\hline 58 & Nižný Slavkov & $\begin{array}{l}\text { banks of Kunišovský potok creek } \\
\text { with Myricaria }\end{array}$ & $\mathrm{A}$ & 49.0920 & 20.8232 & $530-570$ & 13.07.2017 \\
\hline 59 & Petruška saddle & $\begin{array}{l}\text { clearings and meadows in mixed } \\
\text { forest }\end{array}$ & M & 49.0541 & 20.8265 & 820 & 12.07.2017 \\
\hline 60 & Brezovica - Kamenná & $\begin{array}{l}\text { mesic meadow with spring, } \\
\text { abandoned fields }\end{array}$ & M & 49.1245 & 20.8007 & 780 & 30.08 .2017 \\
\hline 61 & Brezovica - Banská & mezic grassalnd in mixed forest & $\mathrm{M}$ & 49.1192 & 20.8001 & 800 & 30.08 .2017 \\
\hline 62 & Brezovica - Šlamov & $\begin{array}{l}\text { montane meado on ridge in } \\
\text { spruce forest }\end{array}$ & $\mathrm{C}$ & 49.1124 & 20.7997 & 900 & 30.08 .2017 \\
\hline
\end{tabular}




\begin{tabular}{|c|c|c|c|c|c|c|c|c|c|c|c|c|c|c|c|c|c|c|c|c|c|c|c|c|c|}
\hline 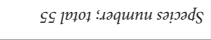 & 2 & $\infty$ & 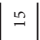 & 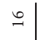 & ¿ิ & \pm & \pm & $\stackrel{\infty}{-}$ & $\stackrel{\infty}{=}$ & ส & \pm & 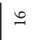 & 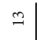 & $=$ & הి & $\simeq$ & $\stackrel{\infty}{-}$ & $\overrightarrow{\mathrm{N}}$ & $\stackrel{\infty}{\sim}$ & $\stackrel{\infty}{=}$ & $\infty$ & 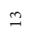 & 6 & $\stackrel{2}{z^{2}}$ & $\begin{array}{ll}2 \\
m \\
m\end{array}$ \\
\hline 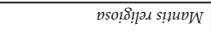 & - & & & & & & & & & & & & & & & & & & & & & & & - & N \\
\hline 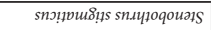 & & & & & & & & & & & & & & & & & & & & & & & & 0 & $\circ$ \\
\hline 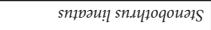 & & & & & a & & N & $m$ & $\sim$ & m & & & & & N & N & $m$ & m & $N$ & $\sim$ & & & & 2 & P \\
\hline 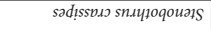 & & & & & & & & & & & & & & & & & & & & & & & & 0 & $\circ$ \\
\hline ! $/$ \&vu vus!podopnas s $_{d}$ & & & & & & & & & t & & & & & & & & & & & & & & & $m$ & 6 \\
\hline 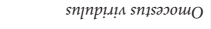 & & & & & & & & & t & a & & - & - & & $\mathrm{a}$ & & & a & & $m$ & & a & & ה & 유 \\
\hline sadynu snısว & & & & & & & & & & & & & & & & & & & & - & & & & a & + \\
\hline 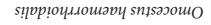 & & & & & - & m & & & & & & & & a & & & & & m & & & & & $n$ & 는 \\
\hline 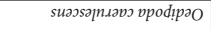 & & & & & & & & & & & & & & & & & $a$ & & & & & & & - & 4 \\
\hline 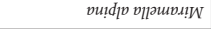 & & & & & & & & & & & & & & & & & & & & & & & & o & $\circ$ \\
\hline 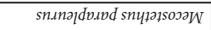 & & & & & & & & & & & & & & & & & & - & & & & & & a & + \\
\hline snfnu snddüгочdиог) & $a$ & & a & $H$ & & - & & & & m & & a & & & & & & $m$ & & & & & & 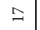 & $m$ \\
\hline 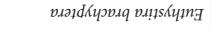 & m & n & m & H & m & m & H & $m$ & t & m & $\sim$ & H & m & m & m & m & $m$ & m & m & m & a & m & - & \&্ & 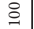 \\
\hline 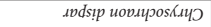 & m & n & m & H & a & - & a & m & 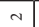 & $\sim$ & $\sim$ & n & m & $\sim$ & $m$ & a & - & m & N & N & $a$ & m & & fo & ஃ \\
\hline suv80 s sndd! & & & & & & & & & & & & & & & & & & & & & & & & - & 4 \\
\hline 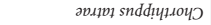 & & & & & & & & & & & & & & & & & & & & & & & & 0 & 0 \\
\hline snllnd sndd! & & & & & & & & & & & & & & & & & & . & & . & & & & - & N \\
\hline 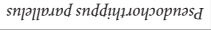 & + & m & m & m & + & $m$ & + & m & t & $\pi$ & $m$ & m & m & & $m$ & $m$ & $m$ & + & + & $\pi$ & m & n & n & 苦 & 2 \\
\hline 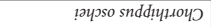 & - & & & & & & & & & & & & & & & & & & & & & & & - & $\mathrm{N}$ \\
\hline 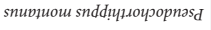 & & & - & & & & & & & & & & a & & & & & & . & & & & & ? & $\overline{4}$ \\
\hline 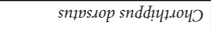 & $t$ & & $\omega$ & - & a & m & & - & $\sim$ & N & $\sim$ & m & & & $m$ & $\sim$ & $m$ & $\sim$ & -1 & $\sim$ & & & & 品 & 8 \\
\hline 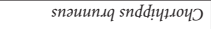 & & & & - & n & $m$ & & & $a$ & & & N & & m & & . & a & & 4 & & & & a & A & 의 \\
\hline 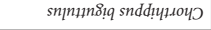 & - & & - & $a$ & 4 & $m$ & & - & $\mathrm{N}$ & $\alpha$ & - & $a$ & & + & - & $m$ & $m$ & & - & & & & $a$ & ¿ & in \\
\hline 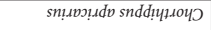 & a & & a & a & a & N & n & a & m & m & $\sim$ & N & a & & m & m & 4 & $\sim$ & & - & & & & $\stackrel{\infty}{m}$ & হ \\
\hline 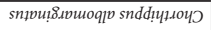 & m & & & & $a$ & & & & & 4 & & & & & & & n & & & & & & & 0 & 2 \\
\hline 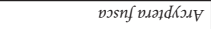 & & & & & & & & & & & & & & & & & & & & & & & & 0 & $\circ$ \\
\hline 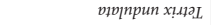 & & & & & & & & & & & - & & & & & & & & & & & & & a & H \\
\hline 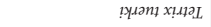 & & & & & & & & & & & & & & & & & & & & & & & $\mathrm{n}$ & $\sim$ & + \\
\hline 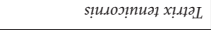 & & & & & & & & & & - & & & & & & & & $\sim$ & - & & & & & 6 & 2 \\
\hline pqvinqns $\times$ xula $L$ & & & & & & & & & & & & & & & & & & & & & & & & 0 & 0 \\
\hline płppundiq xuqa L & & & & & & & & & & & & & & & & & & $a$ & & & & & & - & $\sim$ \\
\hline 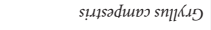 & & & - & & - & & & & & a & & & & & $a$ & & & , & 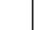 & & & & & $n$ & $\stackrel{2}{2}$ \\
\hline 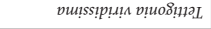 & & & - & & - & & a & - & & - & - & - & & & a & & - & $\sim$ & $\sim$ & $\sim$ & & N & & $\stackrel{2}{2}$ & $\vec{m}$ \\
\hline 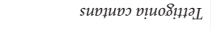 & a & $\sim$ & a & m & $a$ & - & a & 4 & m & $\sim$ & $\mathrm{N}$ & m & m & $n$ & $\sim$ & $\mathrm{n}$ & & $m$ & $m$ & $m$ & $a$ & $m$ & & q & \& \\
\hline 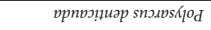 & & & & & & & & & & & & & & & & & & & & . & & & & 4 & 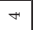 \\
\hline 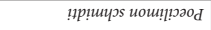 & & a & & - & & & & & & & & & & & & & & $a$ & & $\sim$ & s & & & in & ? \\
\hline 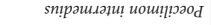 & & & & & & & & & & & & & & & & & & & & & & & & 0 & 0 \\
\hline 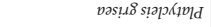 & & & & & - & - & & - & & & & & & & & & a & & & & & & & in & 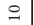 \\
\hline 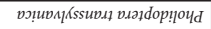 & & m & & a & . & & & . & m & $\sim$ & m & & n & & & & . & 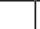 & 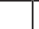 & . & m & m & & $\frac{4}{4}$ & in \\
\hline v.วฺdvoวs!. & $a$ & H & m & th & $a$ & - & a & m & $a$ & $\sim$ & m & 4 & 4 & $n$ & $\sim$ & $\mathrm{N}$ & n & + & m & $m$ & + & t & & J & $\alpha$ \\
\hline 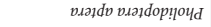 & & & & & & & & & & & & & & & & & & & & & & & & o & $\circ$ \\
\hline 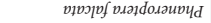 & & & & t & n & & a & m & & $\sim$ & & m & & & m & N & a & m & . & $\sim$ & & & & 2 & o \\
\hline 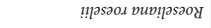 & + & $\sim$ & $m$ & + & a & & + & $m$ & $\sim$ & $\sim$ & $\sim$ & $m$ & $m$ & $a$ & $m$ & $\sim$ & $m$ & + & $m$ & $m$ & $a$ & $m$ & - & \& & इू \\
\hline 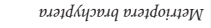 & & & & & & & & & + & & & & & & a & & & & . & & & & & $a$ & 2 \\
\hline solos!q vup.tolos!g & & & & & $a$ & - & a & a & & $a$ & & & & & & & + & & m & & & & & $=$ & ก \\
\hline 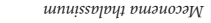 & & & & & & & & & & & & & & & & & & & $\mathrm{N}$ & & & n & & m & 6 \\
\hline 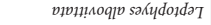 & a & & $m$ & H & m & & m & $m$ & & a & & H & & m & m & 4 & $m$ & + & m & $m$ & & & & ส & to \\
\hline 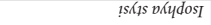 & & & & & & & - & -7 & & . & & & & & - & & & - & & 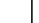 & & & & + & $\infty$ \\
\hline s!suzиə!d phydosI & & & & & & & & - & m & - & - & & N & - & s & & & & & - & & s & & 9 & o \\
\hline !ssnday pরydosI & & & & & & & & & & & & & & & & & & & & $m$ & & & & $m$ & 6 \\
\hline 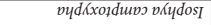 & & & & & & & & & & & & & & & & & & & & & & & & $\circ$ & $\circ$ \\
\hline 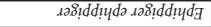 & & & & & & & & & & & & & & & & & & & & & & & & $\circ$ & 0 \\
\hline 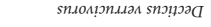 & H & & - & $a$ & m & - & - & m & m & m & - & N & m & $a$ & a & & $m$ & $m$ & m & $m$ & & m & & $\stackrel{\infty}{n}$ & 2 \\
\hline snosnf snppydəวоио J & & & & & & & & & & & & & & & - & & & 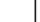 & 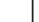 & & & & & - & 4 \\
\hline 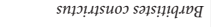 & & & & & & & & & - & & & & - & & & & & - & - & & & - & & n & $\stackrel{20}{7}$ \\
\hline 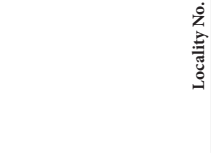 & 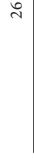 & ง & $\stackrel{\infty}{\sim}$ & ลे & 요 & $\vec{m}$ & $\approx$ & $m$ & m & 柋 & $\infty$ & 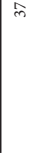 & $\infty$ & के & q & $F$ & F & F & \$ & \& & f & f & $\stackrel{\infty}{+}$ & 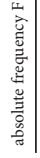 & 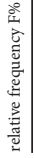 \\
\hline
\end{tabular}




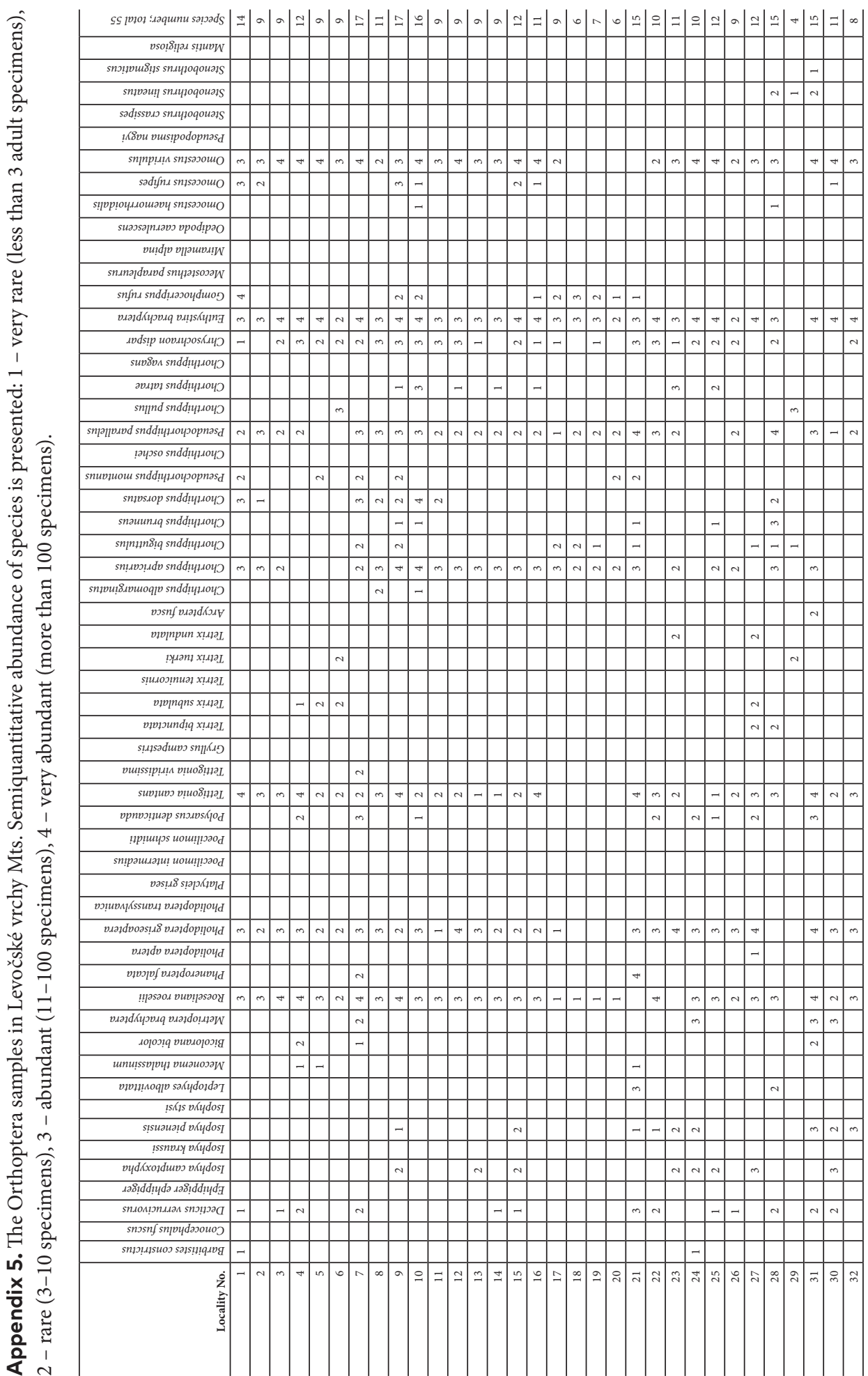




\begin{tabular}{|c|c|c|c|c|c|c|c|c|c|c|c|c|c|c|c|c|c|c|c|c|c|c|c|c|c|c|c|c|c|c|}
\hline 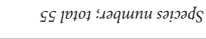 & $\approx 1$ & 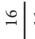 & $\Rightarrow$ & $\infty$ & in & & & & & $\approx=$ & & & & 9 & 이 & $\infty$ & $\simeq$ & $=$ & & $\infty$ & & & $\simeq$ & & $\simeq$ & & & & & $\stackrel{\vec{b}}{\mathrm{\sigma}}$ \\
\hline vsol8?!? s! s!uvW & & & & & & & & & & & & & & & & & & & & & & & & & & & & - & -1 & $\mid 4$ \\
\hline 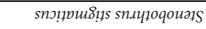 & & & & & & & & & & & & & & & & & & & & & & & & & & & & & - & $\mathrm{a}$ \\
\hline 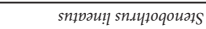 & & & & & & & $\mathrm{N}$ & & & & & & & & & & 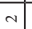 & & $\sim$ & & & & & a & - & & & & $\infty$ & 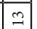 \\
\hline 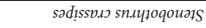 & & & & & & & & & & & & & & & & & & & & & & & $m$ & & & & & & -1 & s \\
\hline 1. $\mathrm{K}$ gvu vusıpodopnәs $d$ & & & & & & & & & & & & & & & & & & & & & & & & & & & & & 0 & $\circ$ \\
\hline 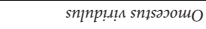 & 4 & & + & & & & m & & & $m a$ & $m-1-1-1-1-1$ & -1 & 4 & 4 & - & & $\sim$ & $H$ & & $\sim$ & $\sim$ & + & & $\sim$ & m & 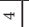 & a & $\mathrm{N} m$ & 尔 & $\stackrel{0}{2}$ \\
\hline 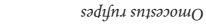 & & & & & & m & & & & & & & & & & & & & & & & & & & & & & & $\infty$ & $m$ \\
\hline 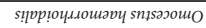 & & & & & & & & & & & & & & & & & & & & & & & & & & - & & & m & in \\
\hline 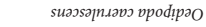 & & & & & & & & & & & & & & & & & & & & & & & & & . & & & & 0 & 0 \\
\hline 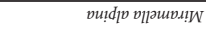 & & & N & & & & & & & & & & & & & & & & & & & & & & & & & & - & $\mathrm{N}$ \\
\hline 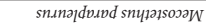 & & & & & & & & & & & & & & & & & & & & & & & & & & & & & 0 & 0 \\
\hline snfnı snddц्әэочdиоо & & & & & & & & & a & & & & & & & & & & $\mathrm{N}$ & & 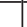 & & & . & 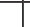 & . & N & & $\approx$ & 2 \\
\hline 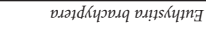 & +4 & $\sim$ & + & & + & $m-1$ & + & $\pi / 2$ & ง & + & H. & $m \mid n$ & $\mathrm{~m}$ & on & m & $m$ & m & H & m & $\sim$ & $m$ & $m$ & $\mathrm{a}$ & $\sim$ & + & m & ง & 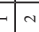 & 8 & 2 \\
\hline ıрds!p иов.ировк.иО & $\mathrm{N}$ & & -1 & & & & m. & $m$ & & $m \mid s$ & $m$ & m & $\omega$ & 4 & - & -1 & $\sim$ & $m$ & $\mathrm{~N}$ & & $\sim$ & + & $a$ & $\mathrm{~N}$ & m & - & - & $\mathrm{a}$ & क & 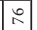 \\
\hline 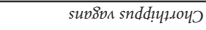 & & & & & & & & & & & & & 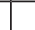 & & & & & & & & & & & 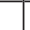 & 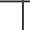 & & & & 0 & $\circ$ \\
\hline 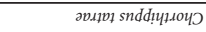 & & & & & & & & & & & & & $a$ & $\mathrm{a}$ & & & & & & & & & & & 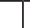 & . & & & $a$ & $\stackrel{2 n}{2}$ \\
\hline 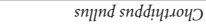 & & m & & & & & & & & & & & & & & & & & & & . & & & 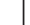 & . & . & 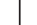 & & m & in \\
\hline 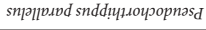 & $\mathrm{AT}$ & $m$ & + & + & & & $m$ & $m$ & $m-1$ & 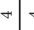 & $A \mid$ & $m$ & $m$ & m & $m$ & $\sim$ & $m$ & $\infty$ & m & $\theta$ & 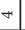 & & $\sim$ & $m$ & + & + & $\mathrm{N}$ & 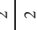 & in & $\infty$ \\
\hline 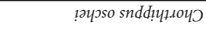 & & & & & & & & & & & & & & & & & & & & & & & 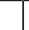 & & 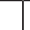 & & & & 0 & 0 \\
\hline 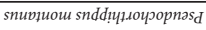 & & & & + & & & 4 4 & n & $\sim$ & 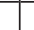 & & & 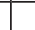 & - & 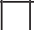 & & $\sim$ & & . & & . & & - & . & 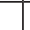 & - & $m$ & & $=$ & $\overline{\mathrm{N}}$ \\
\hline 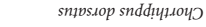 & & & & $N$ & & $m-1-1-1-1-1$ & $-1-$ & - & & $-1-$ & - & & $a$ & $\mathrm{a}$ & - & -1 & $m$ & & $\sim$ & & $\mathrm{N}$ & & $m$ & $\mathrm{n}$ & m & m & - & -1 & 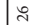 & F \\
\hline 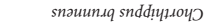 & & 4 & & & & & $m$ & & & & & & & & & & 4 & & & & & & a & 4 & . & & 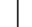 & 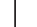 & ㅇ & $\underline{-1}$ \\
\hline 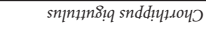 & & 4 & & $m$ & & ( & ง & & & a & ง & & 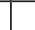 & . & 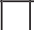 & & $\sim$ & & N & & 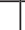 & & $m$ & $\sim$ & - & & -1 & $-m$ & A & m \\
\hline 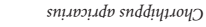 & $\sim$ & + & & - & & $m / 2$ & $\mathrm{~N}$ & & $\omega$ & + & + & m & $m$ & $\mathrm{~m}$ & $m$ & $\sim$ & + & 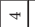 & $m$ & & & & $\mathrm{~m}$ & $\mathrm{a}$ & m & + & 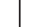 & - & F & $\pi$ \\
\hline 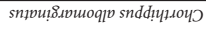 & & & & & & & & & & & & & & & & & & & & & & & & & & & & & 4 & $m$ \\
\hline 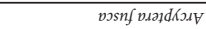 & & & & & & & & & & & & & & & & & & & & & & & & & & & & & - & $\mathrm{n}$ \\
\hline pұvinpun $\times\lfloor$ !1 $L$ & & & & & & & & & & & & & & & & & & & & & & & & & & & & & 4 & m \\
\hline 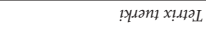 & & $\mathrm{N}$ & & & & & & & & & & & & & & & & & & & & & & 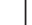 & 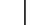 & & & & $m$ & in \\
\hline 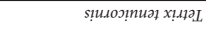 & & $\sim$ & & & & -1 & & & & & & & & & & & & & & & & & & a & -1 & 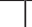 & & & 4 & 6 \\
\hline piplnqns $\times\lfloor$ !u $L$ & & $\mathrm{N}$ & & & & & & & & & & & & & & & & & & & & & & 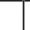 & & - & & & 0 & 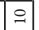 \\
\hline 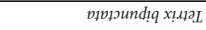 & & $m$ & & & & & & & & & & & - & $-1-$ & & & & & & & . & & & -1 & & & & & 6 & ? \\
\hline s!ulssduw snllix) & & & & & & & & & & & & & & & & & & & & & -1 & & & . & & & & & -1 & $a$ \\
\hline 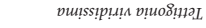 & & & & & & & m 0 & m & & & & & & & & & & & - & & 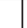 & & 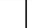 & a & 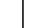 & 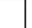 & 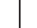 & & H & 6 \\
\hline 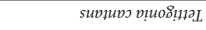 & + & $\mathrm{N}$ & & a 4 & $\sim$ & & - & & & $m$ & 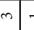 & $-\pi$ & $a$ & $\mathrm{~m}$ & $\omega$ & $a$ & $m$ & $m$ & $\sim$ & $\sim$ & N & m & $a$ & $\sim$ & m & $\sim$ & $m / 2$ & 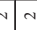 & in & $\mid$ \\
\hline ррпвэ!ฺ & & & & & $m-1-1-1-1-10$ & + & & & & 4 & m & & & & & $m$ & & & & & & & - & & & & & & $\exists$ & m \\
\hline !ฺр!ичрs иот!! & & & & & & & & & & & & & & & & & & & & & & & & & & & & & 0 & 0 \\
\hline 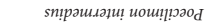 & & & & & & & - & & & & & & & & & & & & & & & & & & & & & & - & a \\
\hline 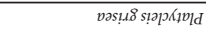 & & & & & & & & & & & & & & & & & & & & & & & & & & & & & 0 & o \\
\hline 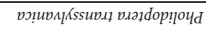 & & & & & & & & & & & & & & & & & & & & & 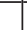 & & 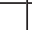 & 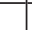 & 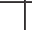 & . & 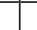 & & 0 & 0 \\
\hline 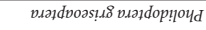 & $m$ & $\sim$ & $a / 2$ & $\sim$ & & & - & & & & $m$ & m & 4 & H & $m$ & & -1 & $a$ & $m$ & $m$ & $m$ & $m$ & $m$ & $\sim$ & m & m & - & 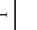 & F & 2 \\
\hline 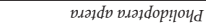 & & & & & & & & & & & & & & & & & & & & & - & & & 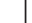 & . & & & & -1 & $\mathrm{a}$ \\
\hline 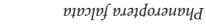 & & - & & & & & & & & & & & & & & & & & & & $\mathrm{N}$ & & 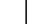 & $\mathrm{a}$ & m & 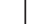 & 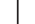 & & 6 & \\
\hline 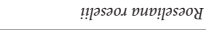 & 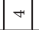 & 4 & 4 a & ง & -8 & $m-1$ & $\pi$ H & $m-1$ & -78 & $m$ & $m$ & $4 / 4$ & $\mathrm{~m}$ & m & $m$ & & $m$ & $m$ & m & $m$ & m & + & 4 & $m$ & + & $\pi$ & a 2 & $4 / 4$ & in & J \\
\hline 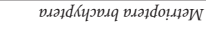 & & & 4 & & & & + & & & $-1-$ & -7. & a & & & & & $m$ & & & & & & 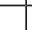 & . & & & - & $-1-$ & I & 2 \\
\hline solos?q pup.opo?tg & & & -1 & & & & + & & & & & & & & & & & & ง & & & & $\sim$ & 4 & & & & & $\infty$ & 2 \\
\hline ипи!sspрұұ ризаоәә & & & & & & & & & & & & & & & & & & & -1 & -1 & . & & 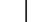 & 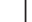 & 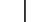 & & & & in & $\left.\right|_{\infty}$ \\
\hline pұvi!n & m & $m$ & & & & -1 & & & & & & & $a$ & a 4 & & & & & m & & $m$ & $m$ & $\mathrm{~m}$ & $\mathrm{a}$ & $m$ & & & & $=$ & $\overrightarrow{4}$ \\
\hline 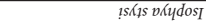 & & & & & & & & & & & & & & & & & & & & & . & & & & & & & & 0 & 0 \\
\hline 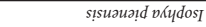 & $m$ & & & & & & & & & & & 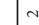 & $\sqrt[n]{4}$ & $\mathrm{~N} / \mathrm{n}$ & & & -1 & $\mathrm{at}$ & $\sim$ & -1 & a & & & & & & & & $\infty$ & 2 \\
\hline Issndxy pKYdosI & & & & & & & & & & & & & & & & & & -1 & & & - & & & & 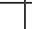 & 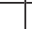 & & & -1 & 4 \\
\hline 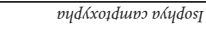 & -7 & & & & & & & & & -- & - & & $a$ & $\mathrm{~N} / \mathrm{n}$ & & & & & & & $\sim$ & & & 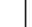 & & & & & \pm & 2 \\
\hline . & & & & & & & & & & & & & & & & & & & 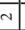 & & - & 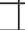 & 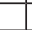 & 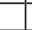 & 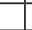 & 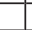 & 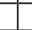 & & -1 & $\mathrm{~N}$ \\
\hline 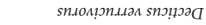 & -1 & -1 & + & & 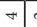 & 4 & $m$ m. & $-1-$ & -10 & \begin{tabular}{c|c}
$\mathrm{a}$ \\
$\mathrm{a}$
\end{tabular} & ง & 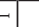 & 4 & \begin{tabular}{c|c|c|}
4 & 4
\end{tabular} & - & 4 & $m$ & $m$ & a & & $\sim$ & -1 & 4 & m & 4 & m & 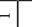 & - & के & 8 \\
\hline snosnf snjpqdəзоио & & & & & & & & & & & & & & & & & & & & & & & & & & & 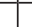 & & 0 & 0 \\
\hline 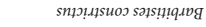 & & & -1 & & & & & & & & & n & & 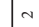 & & & & & -1 & & . & & 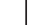 & 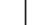 & . & 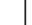 & 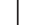 & 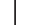 & 6 & ㅇ \\
\hline & & $\vec{m}$ & 紫 & 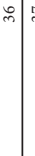 & m. & $\infty$ & $m$ & o $1=$ & $F$ & 7 & $q$ & 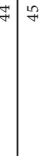 & f & of & $\mid \begin{array}{c}\infty \\
+\end{array}$ & की & in & in & in & in & in & in & in & in & 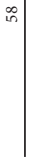 & in & & 68 & 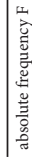 & 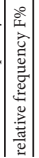 \\
\hline
\end{tabular}

